\title{
Significados asociados al concepto "escuela" de estudiantes en zonas de alta vulnerabilidad social*
}

\author{
Meanings that Students from High Social Vulnerability \\ Areas Relate to the Concept "School"
}

RESUMEN

Se analizan significados atribuidos al concepto escuela por estudiantes de alto rendimiento, en riesgo y de reingreso de educación media superior, inscritos en planteles ubicados en zonas de alta vulnerabilidad social. Las preguntas de investigación fueron: ¿Qué significados atribuyen estudiantes de educación media superior al concepto escuela? ¿Existen diferencias en los descriptores utilizados para definir el concepto escuela entre estudiantes de alto rendimiento, en riesgo y de reingreso? Como técnica para la recolección de información se aplicó un cuestionario semiestructurado. Para analizar la información se obtuvieron los porcentajes de los descriptores y se agruparon en categorías. Los resultados muestran diferencias dependiendo del grupo de referencia de los participantes. Una diferencia cuantitativa se observa al comparar el número de descriptores señalados por los tres grupos. Otra, de naturaleza cualitativa se aprecia al contrastar los tipos de descriptores. En todos los casos, el concepto escuela muestra una valoración positiva y esperanzadora para los jóvenes. Un reto pendiente para la educación formal es empatar las actividades escolares con las expectativas de los jóvenes, con lo cual probablemente se abonará a problemas que aquejan a la media superior en nuestro país, como son el abandono escolar, la reprobación y la eficiencia terminal.

Palabras clave: escuela, educación de jóvenes, representaciones mentales

Gabriela de la Cruz Flores**

\section{ABSTRACT}

The meanings attributed to the concept "school"are analyzed by high performance, at risk and return students of higher education, enrolled in schools located in areas of high social vulnerability. The research questions were: What meanings do high school students attribute to the concept "school"? Are there differences in the descriptors used to define such concept among high performance, at risk and re-entry students? As a technique for collecting information, a semi-structured questionnaire was applied. To analyze the information, the percentages of the descriptors were obtained and grouped into categories. The results show differences depending on the reference group of the participants. A quantitative difference is observed when comparing the number of descriptors indicated by the three groups. Another difference of qualitative nature is appreciated when contrasting the types of descriptors. In all cases, the concept "school" shows a positive and hopeful appreciation for young people. A pending challenge for formal education is to tie school activities with the expectations of young people, which will probably pay to problems that afflict the upper secondary school level in our country, such as school dropout, failure and completion rate.

Key words: school, youth education, expectations, mental representations

*Investigación realizada gracias al Programa UNAM-DGAPA-PAPIIT. Proyecto IN300116 "Equidad educativa: procesos de inclusión-exclusión en Educación Media Superior". Una primera versión del artículo se presentó en el XIV Congreso Nacional de Investigación Educativa organizado por el Consejo Mexicano de Investigación Educativa (COMIE, A.C.).

** Instituto de Investigaciones sobre la Universidad y la Educación (IISUE), UNAM, México; gabydc@unam.mx 


\section{INTRODUCCIÓN}

El presente artículo se deriva de una línea de investigación sobre inclusión-exclusión en educación media superior. La indagación de naturaleza cualitativa, ha consistido en desentrañar prácticas y procesos de inclusión-exclusión en planteles escolares de educación media superior pertenecientes al bachillerato general, universitario y al profesional técnico ubicados en zonas de alta vulnerabilidad social de la Ciudad de México. De acuerdo con Pizarro (2001), con el concepto de vulnerabilidad social se pretende enfatizar las condiciones de vida que, de manera recurrente, colocan a los sujetos en situaciones de desventaja, inseguridad e indefensión ejerciendo parcialmente sus derechos como ciudadanos plenos. Las Alcaldías y colonias donde se ubican los planteles seleccionados tienen en común pertenecer a zonas de nivel socioeconómico bajo, altos niveles de violencia y delincuencia, mercado laboral informal, familias desintegradas, adicciones y narcomenudeo, entre otras.

La investigación se integra por dos fases de trabajo complementarias. La primera, de corte empírico, se ha implementado bajo la lógica de la fenomenología y la etnometodología. La segunda se orienta al trabajo de intervención educativa bajo los supuestos de la investigación-acción cooperativa, en pro de generar condiciones más justas y equitativas que promuevan mejores experiencias educativas en favor de la calidad del aprendizaje.

En las siguientes páginas se presentan algunos resultados derivados de la primera fase. Como punto de arranque se indagaron los significados asociados al concepto escuela de estudiantes inscritos en cuatro planteles públicos que ofrecen formación profesional técnica. Para la selección de los participantes se especificaron criterios que permitieran delimitar diferentes perfiles con base en el rendimiento escolar. El estudio de corte comparativo tuvo como propósito explorar posibles diferencias en los significados atribuidos al concepto escuela, dependiendo del rendimiento escolar de aquéllos. Sin embargo, los resultados permiten apreciar que los jóvenes, independientemente de su rendimiento, mantienen una valoración altamente positiva y esperanzadora sobre el papel de la escuela. Estos hallazgos exigen redoblar con equidad la labor escolar y la política educativa para corresponder a las expectativas 
y representaciones de los jóvenes. Lo expuesto en este párrafo se desarrolla a detalle en las siguientes páginas.

El escrito se divide en cuatro apartados. En el primero se describen algunos antecedentes que enmarcan la indagación, para ello se examinan reportes de investigación que han explorado valoraciones de jóvenes sobre el papel de la escuela y la educación en América Latina. Posteriormente, se analizan estudios que han examinado significados sobre el concepto escuela desde el punto de vista de jóvenes estudiantes. En el segundo apartado, se puntualiza el encuadre metodológico del presente estudio de carácter descriptivo y comparativo. Cabe señalar que se optó por un muestreo deliberado donde participaron estudiantes con perfiles académicos diferenciados: alto rendimiento, en riesgo y de reingreso. Como técnica para la recolección de datos se diseñó un cuestionario semiestructurado. En la tercera parte se presentan los resultados. Para ello se contrasta el número de descriptores para definir el concepto escuela y se agrupan en categorías de análisis. Finalmente, a manera de cierre, se concluye con una serie de implicaciones de los hallazgos y futuras líneas de investigación e intervención educativa, en pro de generar condiciones más justas para todos los jóvenes, en especial para aquellos cuyos entornos sociales se muestran signados por la desigualdad.

\section{ANTECEDENTES}

En América Latina -en las últimas décadas de manera consistente- se han desarrollado políticas que garantizan doce años mínimos de formación escolar. Con la obligatoriedad de la educación media, se pretende aportar al desarrollo social y económico de las naciones, así como transitar hacia modelos educativos inclusivos, dejando atrás su carácter selectivo como un filtro para acceder a la educación superior. Trucco (2014) apoyándose en información de la Comisión Económica para América Latina y el Caribe (CEPAL), advierte sobre la relevancia de la conclusión de la educación media, como se expresa en la siguiente cita:

Es crucial no sólo para adquirir las destrezas básicas que requiere un mundo globalizado y democrático y que permiten al sujeto desenvolverse li- 
bremente y con capacidad para aprender por el resto de su vida. También es determinante para acceder a niveles mínimos de bienestar que le permiten romper los mecanismos de reproducción de la desigualdad que, de lo contrario, afectarán a sus hijos (Trucco, 2014, p. 9).

Sin embargo, la obligatoriedad de la educación media ha dejado al descubierto problemáticas de alto impacto como la baja eficiencia terminal, la reprobación, la repitencia, la sobreedad y el abandono escolar, que minan el pleno derecho a la educación y aquejan con mayor severidad a los sectores sociales más desfavorecidos, ensanchando las brechas de desigualdad y la falta de equidad (Román 2009, 2013). Descuidar o ignorar la atención de los jóvenes de los sectores más desfavorecidos, como lo refiere Villa (2014), incrementa la inequidad del sistema, por lo que subraya la importancia de brindar oportunidades educativas reales e implementar servicios diversificados y justos que atiendan las múltiples circunstancias en que aquéllos se desenvuelven.

Los jóvenes son una población altamente vulnerable de exclusión (Krichesky et al., 2005), lo cual se incrementa cuando, además, pertenecen a sectores desfavorecidos. En 2012 la UNESCO, dando seguimiento al Marco de Acción de Dakar sobre la Educación para Todos (EPT), publicó el informe titulado "Los jóvenes y competencias: Trabajar con la educación", donde se reconoció que los que han crecido en condiciones de pobreza y exclusión tienen más probabilidades de cursar pocos estudios o de abandonar su formación. Conclusiones similares se han obtenido con otros estudios, por ejemplo, los encabezados por Reimers (2002), donde se ha identificado que un sector importante de jóvenes que abandonan los estudios proviene de familias con bajo capital cultural y social, capitales que tienden a no coincidir e incluso contraponerse con las propias demandas y dinámicas escolares. En este sentido, se generan ciclos de exclusión y desigualdad entre la formación y el mercado laboral; es decir, a menor formación (ya sea inconclusa o poco pertinente) mayor marginación laboral. Por ello, desde un enfoque de equidad educativa es imprescindible comprender qué acceso tienen los jóvenes desfavorecidos al desarrollo de competencias que les permitan mejores empleos y romper con condiciones de pobreza. 
En México, Solís (2014), haciendo uso de la encuesta sobre trayectorias educativas y laborales de los jóvenes de la zona metropolitana de la Ciudad de México, en 2010, identificó que sólo $45.7 \%$ de los jóvenes de estrato muy bajo ingresó a la educación media y apenas $23.9 \%$ la terminaron; en contraste, $93.8 \%$ de jóvenes pertenecientes al estrato alto ingresó, y concluyó $76.5 \%$. Además, la desafiliación a la educación media pareciera estar relacionada con el origen social, el apoyo y las expectativas familiares sobre el aporte de la educación. Por ello, hay mayor probabilidad de que la permanencia, la progresión y la conclusión de los estudios sea menor en los jóvenes de los estratos sociales más bajos, problemática que acentúa la relación entre pobreza, marginación y desigualdad educativa. De ahí la importancia de instrumentar acciones que focalicen con equidad la atención de jóvenes de estratos sociales bajos, pues sólo así se podrán romper los ciclos de desigualdad generacional.

De manera paradójica, el carácter incluyente de la educación media superior ha expuesto las debilidades de los sistemas educativos en América Latina como la fragmentación de los aprendizajes entre los estratos sociales más desfavorecidos, aumentando las probabilidades de marginación social y económica (Trucco, 2014). Esta condición ha sido descrita como desigualdad endógena del sistema educativo, la cual hace referencia a la incapacidad de éste para favorecer el aprendizaje y el desarrollo de competencias en los jóvenes para integrarse plenamente a la sociedad, amplificando su efecto en los estratos sociales más bajos. Al respecto Tapia y Valenti (2016) advierten cierta contradicción entre el incremento en la cobertura educativa y la desigual distribución de los aprendizajes, problemática palpable en los resultados diferenciados de pruebas estandarizadas nacionales e internacionales.

Aunado a lo anterior, pareciera que prevalece cierto desencuentro entre los ámbitos, culturas y realidades en que se desarrollan los jóvenes y los sistemas educativos tradicionales, encuadrados en esquemas escolarizados ajenos e incluso en constante tensión con las propias expectativas y necesidades de formación en un mundo complejo, incierto e incluso violento por el que transitan los jóvenes. Contreras y Lafferte (2017:56) señalan que en la actualidad se estima: 
La presencia de jóvenes que descreen de la escuela. En apoyo de esta idea, las encuestas nacionales sobre juventud realizadas en los países de la región (con su variabilidad metodológica y de ciertos aspectos locales) ponen en evidencia la persistencia de los modelos tradicionales de valoración del rol de la escuela, a la vez tensionados por numerosas problemáticas, como el aumento de las brechas educativas según los estratos socioeconómicos, la violencia física y psicológica, y los desajustes entre los aprendizajes y el mercado laboral, entre otros factores.

En estas coordenadas resulta de vital importancia adentrarse en las experiencias y significados que los jóvenes atribuyen a la escuela como institución que social y culturalmente sigue legitimando el rol de la educación formal. Si bien un peso importante de dichos significados corresponde a un legado histórico arraigado a un dispositivo -apelando al concepto de Foucault- cabría cuestionar su vigencia y su configuración en las nuevas generaciones, ante el supuesto fracaso de las instituciones educativas enconchadas en prácticas disonantes.

En México, de manera paralela al crecimiento de investigaciones sobre los jóvenes como sujetos de estudio, se han desarrollado líneas que han indagado sus experiencias y vivencias en ambientes escolares como espacios juveniles, donde se desarrollan múltiples procesos como los documentados por Guzmán y Saucedo (2007) y Weiss (2012, 2018). De manera particular, se destacan ciertos estudios que han explorado cómo los jóvenes significan la escuela, cuyos resultados permiten acercarse y comprender parte de los procesos subjetivos y simbólicos asociados a la construcción de trayectorias escolares (Beatle et al., 2010).

Guerra (2000), a través de un estudio cualitativo que usó como técnica la entrevista, indagó los significados del concepto escuela en estudiantes pertenecientes a un tecnológico $(n=21)$ y a un bachillerato universitario $(n=20)$, ambos ubicados en una zona urbana popular del oriente de la Ciudad de México. En cuanto a los significados atribuidos, la autora identificó nueve categorías: 1. Medio para continuar estudios superiores. 2. Espacio que privilegia un estilo de vida juvenil. 3. Exigencia social. 4. Espacio formativo. 5. Posibilidad de superar la condición social o estatus de familia. 6. Medio que posibilita la movilidad eco- 
nómica. 7. Posibilidad para enfrentar la condición de género. 8. Medio para adquirir autoestima y valoración social. 9. Desafío a la posición o valoración negativa de la familia hacia la escuela. Los resultados mostraron distanciamientos relevantes en los significados dependiendo del plantel escolar. Según la autora, las diferencias socioculturales de los participantes y de la propia oferta educativa son detonantes clave para la configuración de significados. Así, por ejemplo, mientras que los estudiantes del bachillerato universitario reconocieron con mayor frecuencia a la escuela como un espacio formativo y de vida juvenil, en contraste, los jóvenes del bachillerato ponderaron a la escuela como un medio de movilidad social y económica, y como una vía para aumentar su autoestima y valoración social.

En un estudio cualitativo con jóvenes de educación secundaria $(n=57)$, Lozano (2005) usó como técnica la entrevista semiestructurada, y encontró que las representaciones de los estudiantes sobre la escuela y la educación se derivan de referentes altamente instituidos y hegemónicos, donde la disciplina, el control y las sanciones hacia aquellos que no se adaptan al sistema escolar son justificadas. Sin embargo, también identificó ciertas rupturas con ideas preconcebidas sobre la escuela, como es la noción de que aquélla garantiza la movilidad social del educando. El autor en cuestión, advierte tensiones en las representaciones de los jóvenes participantes en su estudio, al respecto la siguiente cita:

Se aprecia la influencia y aceptación de estas representaciones heredadas y aceptadas relativamente por los alumnos, cuando se habla de la necesidad de aceptar los valores y disciplina escolar para poder adaptarse en un futuro a la sociedad, pues quien no aprenda desde ese momento a seguir las normas, podrá fracasar en el futuro. Lo cual se ha convertido en un mito cada vez menos creíble, pues se habla de la crisis de la escuela como factor de movilidad social y esto los alumnos comienzan a percibirlo. De ahí que las representaciones mencionadas entre otras sean heredadas, pero no absolutamente compartidas, lo cual se evidencia en su comportamiento resistente en la escuela que aunque no llega a ser consciente y opositor, si está denotando inconformidad con lo que viven (Lozano, 2005, p.7). 
Si bien la indagación de Lozano (2005) se centró en la educación secundaria, llaman la atención sus conclusiones cuando afirma que, cuando los jóvenes llegan a significar de manera positiva a la escuela, lo hacen como reflejo de una representación hegemónica sobre el poder de la escuela como vehículo para la mejora de vida. La mirada desesperanzadora del autor contrasta con perspectivas teóricas y analíticas en las que se reivindica el papel de la escuela como un espacio para la agencia con el aprendizaje y la configuración de espacios para la participación democrática. Un ejemplo al respecto son las "Escuelas para la Justicia Social" coordinadas por el Grupo de Investigación Cambio Educativo para la Justicia Social (GICE), en España (Perines e Hidalgo, 2018).

Otro estudio, también de naturaleza cualitativa, fue el realizado por Guerrero (2006) donde indagó trayectorias escolares y el denominado turning point en estudiantes inscritos en un bachillerato universitario ubicado en el sur de la Ciudad de México. El concepto de turning point hace referencia a momentos críticos y reflexivos que hacen virar la dirección de las acciones. En el caso particular de la investigación en cuestión, la autora recupera la voz de ocho estudiantes con trayectorias similares, a través del análisis de entrevistas narrativas autobiográficas: discontinuidad y eventos detonantes que modificaron sus experiencias de ser estudiantes. Parte de los resultados permiten apreciar que el ejercicio de la libertad con la que se confrontan los estudiantes en este nivel educativo (contrario al régimen académico de la educación secundaria) los obliga a gestionar nuevas responsabilidades y el ejercicio de la autonomía; por ello este nivel educativo puede significar una etapa crítica para la configuración de la identidad, la toma de decisiones e incluso para dar sentido a los estudios. En palabras de la autora:

Los jóvenes de hoy deben encontrar por sí mismos el sentido de los estudios ... en el entendido de que los certificados no garantizan un lugar en un mercado de trabajo que cada vez exige diplomas más especializados. Saben que si bien los estudios no garantizan un empleo, sin ellos la imposibilidad de tenerlo es mayor (Guerrero, 2006, pp. 503-504). 
El estudio de Guerrero deja entrever que los significados atribuidos a la escuela están mediados por la propia subjetividad y experiencias personales, donde los estudios siguen teniendo un peso en el imaginario para la movilidad social y mejores oportunidades, aunque en un entorno altamente competitivo y selectivo.

En el ámbito internacional, encontramos la indagación de Beatle et al. (2010), quienes a través de un estudio cuantitativo transversal indagaron los significados atribuidos a los conceptos estudio y escuela en una muestra por conveniencia de estudiantes de educación media pertenecientes a diferentes años escolares. En dicho estudio participaron 1546 jóvenes de seis escuelas públicas de Buenos Aires. Como técnica para la recolección de datos los autores utilizaron un cuestionario autoadministrado integrado por preguntas sociodemográficas $y$, para explorar los significados con mayor peso asociados al concepto escuela, se especificaron doce ítem con respuesta tipo Likert (con escala de respuesta $1=$ nada de acuerdo a 5=totalmente de acuerdo). Los ítem en cuestión exploraban las siguientes posibilidades que brinda la escuela: 1. Obtener un título para trabajar. 2. Tener una formación básica para continuar estudiando en el futuro. 3. Desarrollar valores. 4. Formarte como persona. 5. Tener una imagen de tu rendimiento escolar. 6. Relacionarte con otros y tener amigos. 7. Lograr una actitud crítica e independiente. 8. Integrarte a la sociedad. 9. Conocerte mejor. 10. Contar con una cultura general. 11. Aceptar las reglas de convivencia, y 12. lograr un reconocimiento social. Los resultados permiten valorar que los jóvenes de primer y tercer año puntearon con medias altas los ítem 1, 2 y 3 . Mientras que los estudiantes de quinto ańo, además de los ítem 1 y 2 , optaron por el ítem 10. En todos los casos llama la atención que los significados atribuidos a la escuela con mayor peso se relacionen con el futuro y una mirada instrumental de la escuela como un medio para alcanzar un fin (trabajar y continuidad con los estudios).

Prado y Esquerdo (2016) en el contexto brasileño abordaron los motivos por los que jóvenes de educación media del último año asisten a la escuela. La muestra por conveniencia integró estudiantes de escuelas públicas y particulares $(\mathrm{n}=2370)$, a quienes se les aplicó un cuestionario estructurado y, posteriormente, se integró un grupo de conversación con estudiantes participantes 
quienes fueron seleccionados de manera aleatoria $(\mathrm{n}=5)$ para dialogar e interpretar los resultados del cuestionario. Los hallazgos mostraron que los mayores porcentajes se tasaron en los siguientes motivos por los que asisten a la escuela: 1. por la relación que guarda la escuela con los valores de los estudiantes; 2 . la escuela es importante para conseguir un trabajo en el futuro, y 3. la escuela es una vía para ingresar a la educación superior. Las autoras concluyen que, si bien las valoraciones hacia la escuela en su mayoría fueron positivas, también se encontraron ciertas críticas sobre el rol de la escuela en la sociedad contemporánea.

En síntesis, la investigación sobre los significados atribuidos a la escuela por jóvenes presenta ciertas coincidencias en valorar el papel de aquélla; sin embargo, también algunos de los trabajos citados han identificado valoraciones de los jóvenes que cuestionan el papel de la escuela en la actualidad.

En la búsqueda de información sobre investigaciones en torno a los significados atribuidos al concepto escuela, no se encontraron trabajos que abordaran de manera particular los significados de estudiantes de educación media superior inscritos en instituciones escolares ubicadas en zonas desfavorecidas; tampoco se identificaron estudios que exploraran diferencias en los significados relacionados con el concepto escuela atendiendo al rendimiento escolar del estudiantado. Lo anterior justifica el objetivo general de la presente indagación: analizar los significados atribuidos al concepto escuela por estudiantes de alto rendimiento, en riesgo y de reingreso de educación media superior inscritos en escuelas ubicadas en zonas de alta vulnerabilidad social. Las preguntas que guiaron la investigación fueron: ¿Qué significados le atribuyen estudiantes de educación media superior al concepto escuela? ¿Existen diferencias en los descriptores utilizados para definir el concepto escuela entre estudiantes de alto rendimiento, en riesgo y de reingreso?

\section{ENCUADRE METODOLÓGICO}

La presente investigación se enmarca en los estudios orientados al análisis de los sentidos y significados (Rodríguez, Gil y García, 1999) que como sujetos sociales asignamos a nuestro entorno 
y realidad, los cuales emanan de experiencias y vivencias. En el caso específico del trabajo expuesto, se buscó indagar el significado que estudiantes del bachillerato profesional técnico otorgan al concepto "escuela". En los significados asociados al concepto "escuela" no sólo encontramos un campo semántico, sino entramados cognitivos que mantienen cierta lógica y sinergia, lo cuales pueden actuar como mediadores del comportamiento, estructuran el pensamiento y la interacción en el ámbito social. Como dispositivo conceptual se apeló al concepto de representación social. Para Mora (2002:7) "una representación social es una modalidad particular del conocimiento, cuya función es la elaboración de los comportamientos y la comunicación entre los individuos"; en otras palabras, son construcciones colectivas presentes en los discursos y prácticas socioculturales. El concepto de representaciones sociales mantiene una fuerte relación con el constructivismo social, en tanto se asume que aquéllas son producto de intercambios e interacciones compartidas. En el caso particular del presente estudio, los significados asociados al concepto escuela pueden ser producto tanto de la interacción cotidiana de los jóvenes con los entornos escolares, como derivarse de discursos y prácticas socioculturales; en cualquiera de los casos, los significados son representaciones compartidas.

\section{Contexto de la investigación}

La indagación se realizó en cuatro planteles de educación media superior orientados a la formación profesional técnica situados en la Alcaldía de Iztapalapa de la Ciudad de México. Los escenarios tienen en común estar ubicados en zonas con bajo índice de desarrollo social. ${ }^{1}$ En el presente estudio se asume cierta asociación entre estructura espacial y condición socioeconómica. Dicha asociación establece diferencias importantes no sólo a nivel espacial-geográfico, sino también en lo simbólico. Al respecto Savarí (2008) señala que en los espacios urbanos la estructura

\footnotetext{
${ }^{1}$ Se consultó el Índice de Desarrollo Social (IDS) propuesto por el Consejo de Evaluación del Desarrollo Social del Distrito Federal (2011). El índice integra seis indicadores: calidad y espacio disponible en la vivienda; acceso a electricidad; bienes durables; adecuación sanitaria; seguridad social y/o servicio médico; rezago educativo. Dicho índice establece cuatro niveles para su medición: muy bajo, bajo, medio y alto.
} 
espacial está asociada con la condición socioeconómica y con la estructura social. Sin embargo, el mismo autor advierte que la segregación urbana no se asocia necesariamente con desigualdad, por lo que es importante avanzar en el terreno interpretativo para explorar sus significados e implicaciones desde el punto de vista de los actores, de ahí la justificación en el presente estudio por adentrarse en los significados de jóvenes que asisten a escuelas ubicadas en zonas que social, cultural e históricamente han sido estigmatizadas como desfavorecidas.

Cabe señalar que, en la Ciudad de México, la oferta profesional técnica representa la opción educativa con menor demanda y con puntajes de ingreso iguales o menores al percentil 10, como se observa de manera recurrente en las convocatorias de ingreso de la Comisión Metropolitana de Instituciones Públicas de Educación Media Superior (COMIPEMS). Pese a los esfuerzos por mejorar el estatus de la educación profesional técnica, en la Ciudad de México sigue imperando cierta valoración negativa en contraste con el bachillerato universitario.

\section{Participantes}

Las muestras fueron no probabilísticas y se optó por un muestreo deliberado. Para la selección de estudiantes en cada plantel se solicitó a las autoridades organizar grupos que cumplieran con los atributos descritos en el cuadro 1 .

CUADRO 1. Participantes y criterios de selección

\begin{tabular}{|ll|}
\hline \multicolumn{1}{|c|}{ Participantes } & \multicolumn{1}{c|}{ Criterios de selección } \\
\hline Estudiantes de alto rendimiento. & Promedio general igual o superior a 9.00 y sin materias reprobadas. \\
\hline Estudiantes en riesgo. & $\begin{array}{l}\text { Promedio igual o menor a 7.50; dos o más materias reprobadas y asistencia } \\
\text { irregular. }\end{array}$ \\
\hline Estudiantes de reingreso. & $\begin{array}{l}\text { Jóvenes que abandonaron temporalmente los estudios y pasado por lo menos } \\
\text { un ciclo escolar retornaron a los planteles. }\end{array}$ \\
\hline
\end{tabular}

Fuente: elaboración propia.

En las tres muestras se buscó que hubiese representatividad por sexo, semestre, turno y carrera cursada por los estudiantes. 
En total participaron 195 estudiantes: 69 de alto rendimiento, 72 en riesgo y 54 de reingreso.

\section{Técnica}

Como técnica para la recolección de información se utilizó un cuestionario semiestructurado, el cual integraba preguntas relacionadas con aspectos sociodemográficos de los participantes y sus familias, así como una serie de preguntas abiertas que tenían como propósito recabar información sobre actividades que realizaban los jóvenes fuera de la escuela, manejo del tiempo libre y significados atribuidos al concepto escuela. Para fines del presente escrito sólo se presenta el análisis de la pregunta abierta: Para ti, ¿qué es la escuela?

\section{Análisis de la información}

Para el análisis de las respuestas a la pregunta: Para ti, ¿qué es la escuela?, se realizaron dos procedimientos complementarios. El primero fue de orden cuantitativo y contempló los siguientes pasos:

1. Armado de una base de datos en el programa SPSS V22 ${ }^{\oplus}$.

2. En cada respuesta se identificaron descriptores clave, los cuales fueron utilizados para organizar el conjunto de respuestas.

3. Se obtuvieron los porcentajes de los descriptores por cada muestra.

4. Los porcentajes de los descriptores en cada muestra se graficaron con el propósito de identificar cuáles habían tenido mayor peso, estableciendo como punto de corte cuando la línea de las gráficas adquiría una naturaleza asintótica.

5. Para conjuntar los descriptores con mayores porcentajes, por cada muestra se elaboraron gráficas radiales.

El segundo procedimiento, de orden cualitativo, consistió en:

1. Análisis de cada descriptor a fin de identificar las cualidades de las palabras utilizadas para definir el concepto escuela.

2. Construcción de categorías de análisis emanadas del conjunto de los descriptores. 
3. Clasificación de cada descriptor en las categorías de análisis.

4. Validar la clasificación por juicio de expertos.

\section{RESULTADOS}

Sobre las muestras de estudiantes participantes se destaca lo siguiente. En la muestra de estudiantes de alto rendimiento $(n=69)$, la edad promedio fue 17 años $(s=1.7)$ y $56 \%$ fueron mujeres. En cuanto al promedio de calificaciones $71 \%$ reportó uno de 9.6 a 10 . Por otra parte, $73 \%$ vivían con sus padres y hermanos, mientras que $14 \%$ con su mamá y hermanos. Sobre las actividades laborales de las madres, $40 \%$ se dedicaban al hogar, $33 \%$ se desempeñaban como empleadas, seguidas de $17 \%$ destinadas al comercio y $5 \%$ laboraban como profesionales. Sobre las actividades laborales de los padres 36\% eran empleados, seguidos por $19 \%$ de comerciantes, $11 \%$ de manera respectiva se dedicaban a algún oficio o ejercían como profesionales. En cuanto al nivel de estudios de las mamás se destaca que el mayor porcentaje, correspondiente a 29\%, contaban con estudios de educación media superior, mientras que el porcentaje más alto de estudios de los padres fue de primaria con $27 \%$. En cuanto al tiempo de traslado de casa a la escuela $37 \%$ dedicaba menos de 30 minutos, seguidos por $33 \%$ quienes utilizaban entre 30 minutos y 1 hora.

En el caso de los estudiantes de bajo rendimiento $(n=72)$, su edad promedio fue de 17.5 años $(s=1.46)$. Fueron hombres $58 \%$ del total de los participantes. El mayor porcentaje, correspondiente a $25 \%$, señaló contar con un promedio de calificaciones de 7.0 a 7.5. Vivía con sus papás y hermanos $67 \%$, seguidos de $17 \%$ que reportaron vivir con su madre y hermanos. En cuanto a las actividades laborales de los padres, $40 \%$ de las madres se dedicaban al hogar, mientras que $36 \%$ se desempeñaban como empleadas. Sobre las actividades laborales de los papás, los mayores porcentajes fueron representados por empleados (36\%) y oficios (27\%). Según los datos recuperados el mayor porcentaje de madres (43\%) y padres (34\%) contaba con estudios de secundaria. En cuanto al tiempo de traslado de casa a la escuela, $43 \%$ utilizaban entre 30 minutos y 1 hora, seguidos de 29\% que ocupaban menos de 30 minutos. 
Sobre la muestra de estudiantes de reingreso $(n=54)$, su edad promedio fue de 18.4 años ( $s=1.36$ ). Fueron hombres $59 \%$ del total de los participantes. El mayor porcentaje, correspondiente a 38\%, señaló contar con un promedio de calificaciones de 7.6 a 8.00 . Vivía con sus padres y hermanos $71 \%$, seguidos de $20 \%$ que reportaron que vivían con su madre y hermanos. En cuanto a las actividades laborales de los padres, $42 \%$ de las madres se dedicaban al hogar, mientras que 39\% eran empleadas. Sobre las actividades laborales de los padres, los mayores porcentajes fueron representados por oficios (24\%) y empleados (22\%). En cuanto a los estudios de los padres de familia, en el caso de las madres se encontró que $29 \%$ contaba con educación secundaria y otro $29 \%$ con estudios de media superior. Lo anterior se repite en el caso de los estudios de los padres; así, 25\% contaba con formación secundaria y el mismo porcentaje con estudios de media superior. En cuanto al tiempo de traslado de su casa a la escuela, se destaca que $48 \%$ utilizaba menos de 30 minutos, seguido por $45 \%$ quienes reportaron tiempos de traslados entre 30 minutos y 1 hora.

En torno a la pregunta: Para ti ¿qué es la escuela? se presentan los resultados cuantitativos y posteriormente la agrupación de los descriptores por categorías.

\section{Descriptores sobre el concepto escuela: análisis cuantitativo}

Los estudiantes de alto rendimiento refirieron 32 descriptores distintos (ver cuadro 2).

Los porcentajes de los descriptores se graficaron en un histograma para identificar aquéllos con mayor peso, estableciendo como punto de corte cuando la línea de la gráfica adquiría una naturaleza asintótica. Posteriormente, se seleccionaron los descriptores con mayor porcentaje. A manera de ejemplo se muestra el cuadro 3, donde en la primera columna se señalan los descriptores con mayor peso sobre el concepto escuela referidos por la muestra de estudiantes de alto rendimiento; en la segunda se señalan los porcentajes; en la tercera la conversión con el valor más alto tasado en 100 y en la última la inversión de valores, estos últimos se utilizaron para elaborar gráficas radiales. Este proceso 
permite apreciar cuáles son los descriptores que se acercan al centro de la gráfica, con lo cual se busca representar los descriptores que son más cercanos al concepto escuela.

CUADRO 2. Total de descriptores sobre el concepto escuela: estudiantes de alto rendimiento

\begin{tabular}{|c|c|c|c|c|c|}
\hline No. & Descriptores & $\%$ & No. & Descriptores & $\%$ \\
\hline 1 & Un lugar para aprender/formar. & $17.7 \%$ & 17 & $\begin{array}{l}\text { Su función es enseñar capacidades y } \\
\text { habilidades. }\end{array}$ & $1.6 \%$ \\
\hline 2 & $\begin{array}{l}\text { Oportunidad para ser alguien } \\
\text { en la vida. }\end{array}$ & $7.3 \%$ & 18 & $\begin{array}{l}\text { Un lugar donde puedes ejercer } \\
\text { conocimientos. }\end{array}$ & $1.6 \%$ \\
\hline 3 & $\begin{array}{l}\text { Recurso/institución que brinda } \\
\text { información/conocimiento. }\end{array}$ & $7.3 \%$ & 19 & Un lugar donde haces amistad. & $1.6 \%$ \\
\hline 4 & Formación para el futuro. & $7.3 \%$ & 20 & $\begin{array}{l}\text { Permite continuar con la vida } \\
\text { académica. }\end{array}$ & $1.6 \%$ \\
\hline 5 & Nos prepara para el trabajo. & $7.3 \%$ & 21 & Un lugar bueno. & $0.8 \%$ \\
\hline 6 & $\begin{array}{l}\text { Es un lugar/medio de } \\
\text { convivencia/socialización. }\end{array}$ & $5.6 \%$ & 22 & Nos brinda una carrera/título. & $0.8 \%$ \\
\hline 7 & $\begin{array}{l}\text { Un lugar para desenvolvernos } \\
\text { como alumnos y ciudadanos. }\end{array}$ & $4.8 \%$ & 23 & Un lugar para expresarnos. & $0.8 \%$ \\
\hline 8 & $\begin{array}{l}\text { Medio de superación } \\
\text { profesional/personal. }\end{array}$ & $4.8 \%$ & 24 & $\begin{array}{l}\text { Ayuda para desarrollar un proyecto } \\
\text { de vida. }\end{array}$ & $0.8 \%$ \\
\hline 9 & Segundo hogar. & $3.2 \%$ & 25 & Un lugar para desarrollar autonomía. & $0.8 \%$ \\
\hline 10 & $\begin{array}{l}\text { Nos ayuda a ser mejores } \\
\text { personas. }\end{array}$ & $3.2 \%$ & 26 & Ayuda a enfrentar problemas diarios. & $0.8 \%$ \\
\hline 11 & Un lugar importante. & $3.2 \%$ & 27 & $\begin{array}{l}\text { Espacio en comunidad para desarrollar } \\
\text { habilidades y conocimientos. }\end{array}$ & $0.8 \%$ \\
\hline 12 & $\begin{array}{l}\text { Ofrece una vida mejor/da } \\
\text { opciones. }\end{array}$ & $3.2 \%$ & 28 & $\begin{array}{l}\text { Una herramienta para brindar apoyo } \\
\text { a otros. }\end{array}$ & $0.8 \%$ \\
\hline 13 & $\begin{array}{l}\text { Amplía capacidad para realizar } \\
\text { cosas. }\end{array}$ & $2.4 \%$ & 29 & Un lugar para relajarme. & $0.8 \%$ \\
\hline 14 & $\begin{array}{l}\text { Base para disciplinarnos } \\
\text { (valores y responsabilidad). }\end{array}$ & $2.4 \%$ & 30 & Un reto. & $0.8 \%$ \\
\hline 15 & Nos ayuda a seguir adelante. & $2.4 \%$ & 31 & Te hace sentir bien y seguro. & $0.8 \%$ \\
\hline 16 & $\begin{array}{l}\text { Herramienta para prepararnos } \\
\text { a nosotros mismos. }\end{array}$ & $1.6 \%$ & 32 & Un lugar que me gusta. & $0.8 \%$ \\
\hline
\end{tabular}

Fuente: elaboración propia a partir de datos empíricos. 
CUADRO 3. Descriptores con mayor porcentaje sobre el concepto escuela referidos por estudiantes de alto rendimiento, conversión e inversión de valores

\begin{tabular}{|l|c|c|c|}
\hline \multicolumn{1}{|c|}{ Descriptor } & Porcentajes & Conversión & Inversión \\
\hline Un lugar para aprender/formar. & $17.7 \%$ & 100 & 0 \\
\hline Oportunidad para ser alguien en la vida. & $7.3 \%$ & 41 & 59 \\
\hline $\begin{array}{l}\text { Recurso/institución que brinda información/ } \\
\text { conocimiento. }\end{array}$ & $7.3 \%$ & 41 & 59 \\
\hline Formación para el futuro. & $7.3 \%$ & 41 & 59 \\
\hline Nos prepara para el trabajo. & $7.3 \%$ & 41 & 59 \\
\hline Es un lugar/medio de convivencia/socialización. & $5.6 \%$ & 32 & 68 \\
\hline $\begin{array}{l}\text { Un lugar para desenvolvernos como alumnos y } \\
\text { ciudadanos. }\end{array}$ & $4.8 \%$ & 27 & 73 \\
\hline Medio de superación profesional/personal. & $4.8 \%$ & 27 & 73 \\
\hline Segundo hogar. & $3.2 \%$ & 18 & 82 \\
\hline Nos ayuda a ser mejores personas. & $3.2 \%$ & 18 & 82 \\
\hline Un lugar importante. & $3.2 \%$ & 18 & 82 \\
\hline Ofrece una vida mejor/da opciones. & $3.2 \%$ & 18 & 82 \\
\hline
\end{tabular}

Fuente: elaboración propia a partir de datos empíricos.

Como se observa en la gráfica 1 , se representan los 12 descriptores con el mayor peso en las respuestas de los estudiantes de alto rendimiento. El descriptor central del concepto escuela es "un lugar para aprender/formar", seguidos por los que aparecen a mano derecha hasta concluir con el descriptor "ofrece una vida mejor/da opciones".

Por otra parte, en las respuestas de los estudiantes en riesgo se identificó un total de 24 descriptores distintos sobre el concepto escuela, tal como se muestra en el cuadro 4. 


\section{GRÁFICA 1. Descriptores con mayor peso sobre el concepto escuela:} estudiantes de alto rendimiento

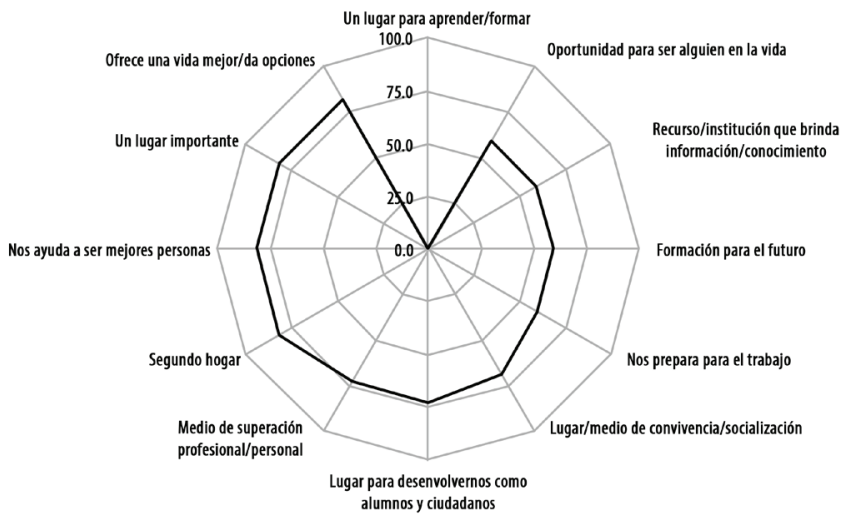

Fuente: elaboración propia a partir de datos empíricos.

Como se observa en la gráfica 2, el análisis permitió identificar un total de siete descriptores con los mayores pesos.

\section{GRÁFICA 2. Descriptores con mayor peso sobre el concepto escuela: estudiantes en riesgo}

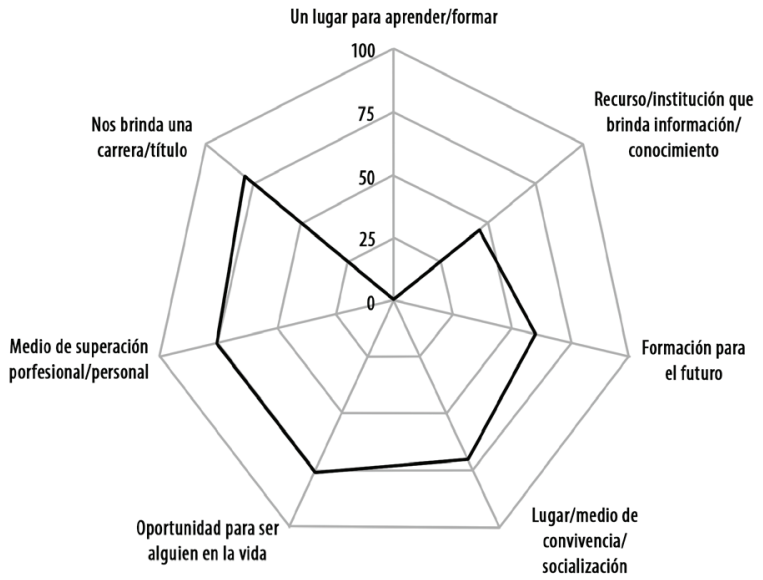

Fuente: elaboración propia a partir de datos empíricos. 
CUADRO 4. Total de descriptores sobre el concepto escuela: estudiantes en riesgo

\begin{tabular}{|c|c|c|c|c|c|}
\hline No. & Descriptores & $\%$ & No. & Descriptores & $\%$ \\
\hline 1 & Un lugar para aprender/formar & $25 \%$ & 13 & Un lugar importante & $1.2 \%$ \\
\hline 2 & $\begin{array}{l}\text { Recurso/institución que brinda } \\
\text { información/conocimiento }\end{array}$ & $13.7 \%$ & 14 & Algo necesario & $1.2 \%$ \\
\hline 3 & Formación para el futuro & $10 \%$ & 15 & Un lugar para relajarme & $1.2 \%$ \\
\hline 4 & $\begin{array}{l}\text { Es un lugar/medio de } \\
\text { convivencia/socialización }\end{array}$ & $7.5 \%$ & 16 & Un lugar que me gusta & $1.2 \%$ \\
\hline 5 & $\begin{array}{l}\text { Oportunidad para ser alguien } \\
\text { en la vida }\end{array}$ & $6.2 \%$ & 17 & $\begin{array}{l}\text { Un lugar donde puedes ser tú } \\
\text { libremente }\end{array}$ & $1.2 \%$ \\
\hline 6 & $\begin{array}{l}\text { Medio de superación } \\
\text { profesional/personal }\end{array}$ & $6.2 \%$ & 18 & $\begin{array}{l}\text { Espacio en comunidad para } \\
\text { desarrollar habilidades y } \\
\text { conocimientos }\end{array}$ & $1.2 \%$ \\
\hline 7 & Nos brinda una carrera/titulo & $5 \%$ & 19 & Una ayuda & $1.2 \%$ \\
\hline 8 & $\begin{array}{l}\text { Función de enseñar } \\
\text { capacidades y habilidades }\end{array}$ & $2.5 \%$ & 20 & La base de los estudios & $1.2 \%$ \\
\hline 9 & $\begin{array}{l}\text { Un lugar donde puedes ejercer } \\
\text { conocimientos }\end{array}$ & $2.5 \%$ & 21 & Nos prepara para el trabajo & $1.2 \%$ \\
\hline 10 & Un centro de educación & $2.5 \%$ & 22 & Un lugar de esparcimiento & $1.2 \%$ \\
\hline 11 & $\begin{array}{l}\text { Ofrece una vida mejor/da } \\
\text { opciones }\end{array}$ & $2.5 \%$ & 23 & Lugar donde haces amistad & $1.2 \%$ \\
\hline 12 & Segundo hogar & $1.2 \%$ & 24 & Nos ayuda a seguir adelante & $1.2 \%$ \\
\hline
\end{tabular}

Fuente: elaboración propia a partir de datos empíricos.

En el cuadro 5 se enlistan 22 descriptores diferentes ubicados en las respuestas de los estudiantes de reingreso.

Se identificó un total de ocho descriptores con mayor peso. $\mathrm{Al}$ igual que en las respuestas de estudiantes de alto rendimiento y en riesgo, el descriptor "un lugar para aprender/formar" obtuvo el mayor peso, como se valora en la gráfica 3 . 
CUADR0 5. Total de descriptores sobre el concepto escuela: estudiantes de reingreso

\begin{tabular}{|c|c|c|c|c|c|}
\hline No. & Descriptor & $\%$ & No. & Descriptor & $\%$ \\
\hline 1 & Un lugar para aprender/formar & $20.5 \%$ & 12 & Un lugar importante & $2.7 \%$ \\
\hline 2 & Nos prepara para el trabajo & $12.3 \%$ & 13 & Ayuda a enfrentar problemas diarios & $1.4 \%$ \\
\hline 3 & $\begin{array}{l}\text { Oportunidad para ser alguien en } \\
\text { la vida }\end{array}$ & $11 \%$ & 14 & $\begin{array}{l}\text { Es un lugar/medio de convivencia/ } \\
\text { socialización }\end{array}$ & $1.4 \%$ \\
\hline 4 & Formación futuro & $6.8 \%$ & 15 & Nos ayuda a seguir adelante & $1.4 \%$ \\
\hline 5 & $\begin{array}{l}\text { Medio de superación profesional/ } \\
\text { personal }\end{array}$ & $6.8 \%$ & 16 & Reforzamiento de cultura e información & $1.4 \%$ \\
\hline 6 & Nos ayuda a ser mejores personas & $5.5 \%$ & 17 & Un lugar de esparcimiento & $1.4 \%$ \\
\hline 7 & Nos brinda una carrera/título & $5.5 \%$ & 18 & $\begin{array}{l}\text { Un lugar donde forjar la vida que } \\
\text { queremos }\end{array}$ & $1.4 \%$ \\
\hline 8 & $\begin{array}{l}\text { Recurso/institución que brinda } \\
\text { información/conocimiento }\end{array}$ & $5.5 \%$ & 19 & Un lugar para desarrollar autonomía & $1.4 \%$ \\
\hline 9 & Ofrece una mejor vida (da opciones) & $4.1 \%$ & 20 & $\begin{array}{l}\text { Un lugar para desenvolvernos como } \\
\text { alumnos y ciudadanos }\end{array}$ & $1.4 \%$ \\
\hline 10 & Amplía capacidad para realizar cosas & $2.7 \%$ & 21 & Un lugar para divertirnos & $1.4 \%$ \\
\hline 11 & Un centro de educación & $2.7 \%$ & 22 & Una herramienta para brindar apoyo a otros & $1.4 \%$ \\
\hline
\end{tabular}

Fuente: elaboración propia a partir de datos empíricos.

\section{GRÁFICA 3. Descriptores con mayor peso sobre el concepto escuela: estudiantes de reingreso}

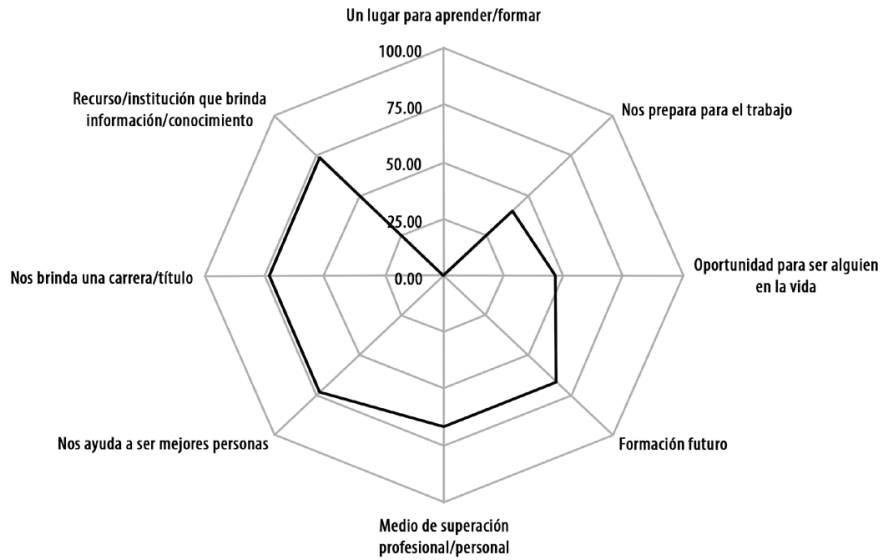

Fuente: elaboración propia a partir de datos empíricos. 


\section{Descriptores del concepto escuela agrupados por categorías: análisis cualitativo}

El conjunto de los descriptores se organizó en cuatro grandes categorías, las cuales fueron validadas por diez jueces con un acuerdo mínimo de $80 \%$. Las categorías fueron:

a) Escuela y vínculo con el futuro.

b) Escuela como espacio de interacción y convivencia.

c) Escuela como entorno de aprendizaje.

d) Escuela como espacio de seguridad y crecimiento.

En la categoría escuela y vinculo con el futuro se agruparon los descriptores: formación para el futuro, nos ayuda a seguir adelante, permite continuar con la vida académica, nos brinda una carrera/título, nos prepara para el trabajo, un lugar donde forjar la vida que queremos y oportunidad para ser alguien en la vida. El conjunto de dichos descriptores tiene en común valorar a la escuela como una entidad que facilitará opciones para el futuro y mejora de las condiciones de vida.

En la categoría escuela como espacio de interacción y convivencia, se agruparon los descriptores: es un lugar/medio de convivencia/ socialización, espacio en comunidad para desarrollar habilidades y conocimiento, lugar donde haces amistad, un lugar de esparcimiento, un lugar para divertirnos, un lugar para expresarnos, un lugar para relajarme y una herramienta para brindar apoyo a otros. Como se aprecia, los descriptores tienen en común significar a la escuela como un espacio social, de encuentro y expresión entre la comunidad escolar.

En la categoría escuela como entorno de aprendizaje, se agruparon los descriptores: amplía capacidad para realizar cosas, ayuda a enfrentar problemas diarios, ayuda a planear un proyecto de vida, base para disciplinarnos, herramienta para prepararnos a nosotros mismos, la base de los estudios, recurso/institución que brinda información/conocimiento, refuerza cultura e información, su función es enseñar capacidades y habilidades, un centro de educación, un lugar donde puedes ejercer conocimientos, un lugar para aprender/formar, un reto, una ayuda. Los descriptores 
en cuestión, hacen referencia a valorar la escuela como un espacio para la formación académica, la promoción de la cultura, el desarrollo de hábitos y habilidades para la vida.

Finalmente, en la cuarta categoría escuela como espacio de seguridad y crecimiento, se agruparon los descriptores: medio de superación profesional/personal, nos ayuda a ser mejores personas, ofrece una mejor vida/da opciones, segundo hogar, un lugar donde puedes ser tú libremente, un lugar para desarrollar autonomía, te hace sentir bien y seguro y un lugar para desenvolvernos como alumnos y ciudadanos. Es de llamar la atención que en esta categoría los descriptores enfatizan la valoración de la escuela como un espacio que aporta al crecimiento personal y profesional de los jóvenes, así como un espacio para la libertad, el desarrollo de autonomía y el aporte a la ciudadanía (reconocimiento de derechos y obligaciones).

\section{DISCUSIÓN Y CONCLUSIONES}

Los resultados expuestos tienen distintas dimensiones analíticas y posibles interpretaciones. Una primera dimensión, de naturaleza social, se refiere a los significados positivos atribuidos al concepto escuela de estudiantes en zonas desfavorecidas. Una interpretación sobre estos resultados podría encontrarse en la configuración de significados altamente instituidos que actúan como un conjunto de creencias resistentes y hegemónicas. Sin embargo, esta posible interpretación minimiza la capacidad de los jóvenes para valorar su entorno y construir significados al respecto. Desde esta mirada constructiva, esperanzadora y cargada de expectativas positivas hacia la escuela se asume que los jóvenes elaboran significados en torno al concepto escuela. Lo anterior también rompe con ciertos estigmas territoriales (Saraví, 2008) para avanzar hacia aproximaciones más comprensivas e interpretativas desde la mirada de los actores. Por otra parte, las valoraciones positivas de los jóvenes hacia el concepto escuela contrastan con estudios donde se asume el desencanto de los jóvenes hacia la escuela y la educación (Contreras y Lafferte, 2017), por lo que conviene ahondar al respecto. 
Si bien los criterios para la selección de las muestras fueron deliberados, llaman la atención algunos datos sociodemográficos que coinciden con otros estudios, por ejemplo, los reportados en la Encuesta Nacional de Deserción en la Educación Media Superior (SEP, 2012). En el caso de la muestra de estudiantes de alto rendimiento, se observa un mayor porcentaje de estudiantes mujeres $(56 \%)$, mientras que en las muestras de estudiantes en riesgo y de reingreso es mayor el porcentaje de hombres $(58 \%$ y $59 \%$ respectivamente), lo cual mantiene cierta relación con la probabilidad de que sean éstos quienes abandonen con mayor frecuencia los estudios en contraste con la mujeres, marcando diferencias de género relevantes en la permanencia y rendimiento escolar que merecen mayor indagación en pro de generar sistemas educativos inclusivos.

Por otra parte, en el caso de la muestra de estudiantes de alto rendimiento, el nivel educativo de las madres con mayor porcentaje fue educación media superior, en contraste con las madres de los estudiantes en riesgo, quienes reportaron un menor nivel escolar, lo cual concuerda con diversos estudios que han identificado el nivel educativo de las madres como un predictor en la continuidad con los estudios.

Sobre las diferencias en los descriptores utilizados para definir el concepto escuela por estudiantes de alto rendimiento, en riesgo y de reingreso, los resultados permiten concluir una diferencia de orden cuantitativa atendiendo el número de descriptores diferentes referidos en cada grupo, donde los estudiantes de alto rendimiento mencionaron un mayor número de descriptores (32), comparados con los referidos por estudiantes en riesgo (24) y estudiantes de reingreso (22). Probablemente las diferencias en cuanto al número de descriptores se deban a que, en el caso de estudiantes de alto rendimiento, éstos atribuyan mayores significados y expectativas al concepto en cuestión o que posean un mejor capital cultural que les permita codificar con un mayor número de palabras. En el caso de los estudiantes de reingreso, probablemente la experiencia de haber estado fuera de la escuela un periodo de tiempo les permitió compactar los significados atribuidos al concepto escuela, por ello, no es errado que el segundo descriptor con mayor peso haya sido "nos prepara para el trabajo". 
Por otra parte, el análisis permitió agrupar en cuatro categorías los descriptores utilizados por los estudiantes, a saber: escuela y vínculo con el futuro; escuela como espacio de interacción y convivencia; escuela como entorno de aprendizaje y escuela como espacio de seguridad y crecimiento. Estas cuatro categorías pueden interpretarse como necesidades de formación, como expectativas en torno a la escuela o como producto de las vivencias y experiencias de los estudiantes. Cabe señalar que estas categorías y sus descriptores guardan una estrecha relación con los reportados por autores referidos con anterioridad (Beatle, 2010; Guerra, 2000; Guerrero, 2006; Prado y Esquerdo, 2016), sin embargo, en el presente estudio la riqueza de los descriptores es más vasta y compleja. De manera particular, la categoría "escuela como un espacio de seguridad y crecimiento" adquiere un significado especial que merece profundizarse, pues es probable que los entornos escolares sean para los jóvenes espacios de resguardo ante una sociedad colapsada por la violencia en que se desenvuelven.

La gran mayoría de los descriptores que utilizaron los tres grupos de estudiantes fueron compartidos; sin embargo, se identificaron descriptores propios en cada grupo. En el caso de los referidos por los estudiantes de alto rendimiento, se destacan: ayuda para desarrollar un proyecto de vida, base para disciplinarnos, herramienta para prepararnos a nosotros mismos, permite continuar con la vida académica, te hace sentir bien y seguro, un lugar bueno, un lugar para expresarnos, un reto. Por su parte, en las respuestas de los estudiantes en riesgo se identificaron los descriptores: algo necesario, la base de los estudios, un lugar donde puedes ser tú libremente y una ayuda. Por último, en las respuestas de estudiantes de reingreso se encontraron los descriptores: reforzamiento de cultura e información, un lugar donde forjar la vida que queremos y un lugar para divertirnos.

La trascendencia del presente estudio radica en centrase en los significados que estudiantes pertenecientes a la formación profesional técnica otorgan al concepto escuela, más aún cuando los participantes asistían a planteles escolares ubicados en zonas de alta vulnerabilidad social, lo cual deja entrever las altas expectativas de este grupo de jóvenes sobre la escuela y el poder de la educación. Un reto pendiente para la educación formal es que las 
actividades escolares empaten con las necesidades y expectativas de los jóvenes, con lo cual probablemente se abonará a problemas que aquejan a la educación media superior en nuestro país, como son el abandono escolar, la reprobación, la repitencia y la eficiencia terminal.

Cabría cuestionarse sobre los significados positivos hacia el concepto escuela que, pese a los magros resultados en pruebas estandarizadas, sigue siendo reconocida y valorada por los jóvenes. También sería importante contrastar los significados atribuidos y las experiencias escolares, con el propósito de articular de manera propositiva desde la intervención educativa mejores prácticas y procesos que atiendan con equidad a los sectores más desprotegidos.

Los resultados expuestos en el presente estudio se limitan exclusivamente a lo que los participantes refirieron por escrito sobre el concepto escuela. En aras de profundizar en los resultados, convendría triangularlos utilizando otros métodos y técnicas para la recolección de información. Una limitante del presente estudio radica en que se centró en los significados del concepto escuela, pero no cuestionó de manera directa sobre los planteles escolares específicos.

Como líneas de investigación convendría explorar los significados del concepto escuela en la gama de opciones y modalidades de la educación media superior. En el caso particular de la formación profesional técnica, sería recomendable replicar la indagación en otros planteles y entidades federativas.

Finalmente, sería recomendable para futuras investigaciones contrastar significados en torno al concepto escuela en planteles escolares ubicados en zonas con distintos grados de vulnerabilidad social, con el propósito de explorar diferencias y semejanzas. Por otra parte, el concepto de vulnerabilidad social sería de alta relevancia para profundizar en los significados que los jóvenes han construido sobre la escuela y la educación en su conjunto.

\section{REFERENCIAS BIBLIOGRÁFICAS}

Beatle, S., Vidondo, M., Kaliman, F., Sansone, C., Nuñez, M., Bory, G., Dueñas, M., Solano, L. y Maldonado, S. (2010). 
El significado del estudio y de la escuela a lo largo de la escuela media. Anuario de Investigaciones, 17, 121-128.

Consejo de Evaluación del Desarrollo Social del Distrito Federal (2011). Indice de Desarrollo Social de las unidades territoriales del Distrito Federal Delegación, Colonia y Manzana. México: Consejo de Evaluación del Desarrollo Social del Distrito Federal.

Contreras, D. y Lafferte, M. (2017). La dimensión subjetiva de los procesos de desescolarización. Debate actual sobre representaciones sociales e identidad en la relación entre los jóvenes y la escuela secundaria. En: López, N., Opertti, R. y Vargas, C. (Coords.). Adolescentes y jóvenes en realidades cambiantes. Notas para repensar la educación secundaria en América Latina, (pp.41-61). Francia: UNESCO.

Guerra, M. (2000). ¿Qué significa estudiar el bachillerato? La perspectiva de los jóvenes en diferentes contextos socioculturales. Revista Mexicana de Investigación Educativa, 5(10), 243-272.

Guerrero, M. (2006). El punto de retorno. Una experiencia de estudiantes de bachillerato universitario. Revista Mexicana de Investigación Educativa, 11(29), 483-507.

Guzmán, C. y Saucedo, C. (2007). La voz de los estudiantes. Experiencias en torno a la escuela. México: Centro Regional de Investigaciones Multidisciplinarias/Facultad de Estudios Superiores Iztacala-UNAM/Ediciones Pomares.

Krichesky, M. Duro, E., Vitar, A., Feldman, D., Croce, A., Kaplan, C., Borzese, D. Bottinelli, L. (2005). Adolescentes e inclusión educativa. Un derecho en cuestión. Buenos Aires: Noveduc.

Lozano, J. (2005). Los significados de los alumnos hacia la escuela secundaria en México. Revista Iberoamericana de Educación, 36(9), 1-9.

Mora, M. (2002). La teoría de las representaciones sociales de Serge Moscovici. Athenea Digital, 2, 1-25.

Perines, H. e Hidalgo, N. (2018). "La escuela confía en que los estudiantes podemos cambiar el mundo": un estudio de las escuelas que trabajan para la justicia social. Revista Colombiana de Educación, 75, 19-38. 
Pizarro, R. (2001). La vulnerabilidad social y sus desafios: una mirada desde América Latina. Chile: CEPAL, Serie Estudios Estadísticos División de Estudios y Proyecciones Económicas.

Prado, B. y Esquerdo, R. (2016). ¿ Por que ir à Escola? Os sentidos atribuídos pelos jovens do ensino médio. Educação e Realidade, 41(1), 93-216.

Reimers, F. (Ed.) (2002). Distintas escuelas, diferentes oportunidades. Los retos para la igualdad de oportunidades en Latinoamérica. España: La Muralla.

Rodríguez, G., Gil, J. y García, E. (1999). Metodología de la investigación cualitativa. España: Ediciones Aljibe.

Román, M. (2009). El fracaso escolar de los jóvenes en la enseñanza media ¿Quiénes y por qué abandonan definitivamente el liceo en Chile? Revista Iberoamericana sobre Calidad, Eficacia y Cambio en Educación, 7(4), 95-119.

Román, M. (2013). Factores asociados al abandono y la deserción escolar en América Latina: una mirada en conjunto. Revista Iberoamericana sobre Calidad, Eficacia y Cambio en Educación, 11(2), 33-59.

Saraví, G. (2008). Mundos aislados: segregación urbana y desigualdad en la Ciudad de México. Eure, 34(103), 93-110.

Secretaría de Educación Pública (2012). Reporte de la Encuesta Nacional de Deserción en la Educación Media Superior. México: SEP.

Solís, P. (2014). Desigualdad social y efectos institucionales en las transiciones educativas. Caminos desiguales. En: Blanco, E. Solís, P. y Robles, H. (Coords.). Trayectorias educativas y laborales de los jóvenes en la Ciudad de México (pp.71-106). México: Instituto Nacional para la Evaluación de la Educación.

Tapia, L. y Valenti, G. (2016). Desigualdad educativa y desigualdad social en México. Nuevas evidencias desde las primarias generales en los estados. Perfiles educativos, 38(151), 32-54.

Trucco, D. (2014). Educación y desigualdad en América Latina. Serie Políticas sociales, Comisión Económica para América Latina y el Caribe, 200, 1-33. 
UNESCO (2012). Informe de Seguimiento de la EPT en el Mundo. Los jóvenes y las competencias: Trabajar con la Educación. Francia: UNESCO.

Villa, L. (2014). La educación media superior, jóvenes y desigualdad de oportunidades. Innovación Educativa, 14(64), 33-46.

Weiss, E. (2018). Los significados del bachillerato para los jóvenes y la permanencia escolar en México. Sinéctica, 51, $1-19$.

Weiss, E. (Coord.) (2012). Jóvenes y bachillerato. México: ANUIES. 